\title{
Ab Initio Electrical Conductance of a Molecular Wire
}

\author{
ROI BAER, ${ }^{1}$ DANIEL NEUHAUSER ${ }^{2}$ \\ ${ }^{1}$ Institute of Chemistry and the Lise Meitner Minerva-Center for Quantum Chemistry, the Hebrew \\ University of Jerusalem, Jerusalem 91904 Israel \\ ${ }^{2}$ Department of Chemistry and Biochemistry, University of California, Los Angeles, CA 90025
}

Received 29 November 2001; accepted 21 May 2002

DOI 10.1002/qua.10449

\begin{abstract}
A method is developed for computing the direct current and alternating current conductance of molecular wires at small bias. The basic ingredients are: linear response theory, time-dependent density functional theory, imaginary potentials, and a jellium model for the metallic leads. The theory is capable of incorporating the effect of realistic charge distributions in the system and electron-electron correlation. We demonstrate the method on the jellium- $\mathrm{C}_{3}$-jellium system. (C) 2002 Wiley Periodicals, Inc. Int J Quantum Chem 91: 524-532, 2003
\end{abstract}

Key words: alternating current; conductance; direct current; jellium model; molecular wire

\section{Introduction}

C an single molecules function as electronic components? This is the central issue addressed by the emerging field of molecular electronics [1]. Even though the first molecular device, a rectifier, was conjectured by Aviram and Ratner [2] as early as 1974, only recently have such systems become accessible to experiments [3-9]. It is difficult to overestimate the impact this field can have. Molecular electronic components are predicted to be smaller and faster than contemporary electronic devices by five to six orders of magnitude [10].

Correspondence to: R. Baer; e-mail: roi.baer@huji.ac.il Contract grant sponsor: Israel Science Foundation.
Spectacular applications of such devices may even include developing machine intelligence competing with humans.

The role of theory in the molecular electronics field is important. Approximate theoretical models are valuable guides to the discovery of new phenomena, therefore pinpointing directions for experimental research. Accurate models on the other hand are indispensable for interpreting intricate experimental data, and they provide high-quality benchmarks for testing and improving the approximations. Yet accurate treatments of the electron dynamics in molecular wires are rare. Most treatments are within the framework of the Landauer formalism [11, 12]. This approach proved especially useful for studying mesoscopic systems $[13,14]$ and forms the basis of several studies of molecular 
wires, based on different levels of electronic structure theory, such as extended Huckel or tight binding [15-21], and tight binding supplemented with self-consistent field electrostatic potential [22] as well as ab initio Hartree-Fock theory [23-25] or density functional theory [26-28].

The ab initio computations within the Landauer framework are high level but they assume fast electron response. Sluggish response and inelastic effects cannot be addressed. The theory is also limited to the direct current (DC) conductance, while alternating current $(\mathrm{AC})$ characteristics of molecular wires are important, as well [29]. It is well established that electronic correlation effects are important for understanding the optical response of molecules. Thus, the role of correlation may also be important in processes of electronic transport in molecules.

In this article we outline a framework in which benchmark-quality AC and DC response of a molecular wire connected to metallic leads can be made. The method is based on linear response theory, time-dependent density functional theory (TDDFT) [30], and a special treatment of the metallic leads. Previous attempts $[31,32]$ to use timedependent functional theory for computing DC conductance relied on a low transmission probability and short time approximation. Such an approach is not always adequate for systems with large conductance.

\section{Conductance by Linear Response}

The AC conductance along the $z$-axis between two points $z_{1}, z_{2}$ is a time-dependent function $G\left(z_{2}\right.$; $\left.z_{1}, t-t^{\prime}\right)$ defined in terms of the current $I\left(z_{2}, t\right)$ induced in a system by a small time and spatial total electric field $E\left(z_{1}, t^{\prime}\right)$ :

$$
I\left(z_{2}, t\right)=\int_{-\infty}^{\infty} \int_{-\infty}^{t} G\left(z_{2} ; z_{1}, t-t^{\prime}\right) E\left(z_{1}, t^{\prime}\right) d t^{\prime} d z_{1}
$$

To compute the conductance, we consider a timedependent Hamiltonian:

$$
\hat{H}_{m b}(t)=\hat{H}_{0}+\sum_{n=1}^{N_{e}} v\left(z_{n}\right) p(t)
$$

where $\hat{H}_{0}$ is the full many-body electronic Hamiltonian and

$$
p(t)=e^{-\left(t-T_{0}\right)^{2} / 2 \sigma^{2}}
$$

is a dimensionless time-dependent envelope function. The perturbation is caused by an electric field $E$ directed along the $z$ axis and spatially localized near $z=z_{0}$. This field is given by:

$$
\mathrm{E}(t, z)=e^{-1} p(t) v^{\prime}\left(z-z_{0}\right)
$$

The electric field induced by transverse currents within the wire is neglected in this treatment. We now define a $z$-averaged conductance:

$$
\bar{G}\left(z_{2} ; z_{0}, t\right)=\Delta v^{-1} \int_{-\infty}^{\infty} G\left(z_{2} ; z, t\right) v^{\prime}\left(z-z_{0}\right) d z
$$

with the total potential difference defined as:

$$
\Delta v=\int_{-\infty}^{\infty} v^{\prime}(z) d z
$$

With this quantity, the conductance is related to the current by:

$$
I\left(z_{2}, t\right)=e^{-1} \Delta v \int_{0}^{\infty} \bar{G}\left(z_{2} ; z_{0}, \tau\right) p(t-\tau) d \tau
$$

After Fourier transforming this expression, we obtain a simple relation between the AC current and the AC conductance:

$$
\tilde{G}\left(z_{2} ; z_{0}, \omega\right)=\frac{e I\left(z_{2}, \omega\right)}{p(\omega) \Delta v}
$$

where the $\mathrm{AC}$ conductance is:

$$
\tilde{G}\left(z_{2} ; z_{0}, \omega\right)=\int_{0}^{\infty} \bar{G}\left(z_{2} ; z_{0}, t\right) e^{i \omega t} d t
$$

and: 


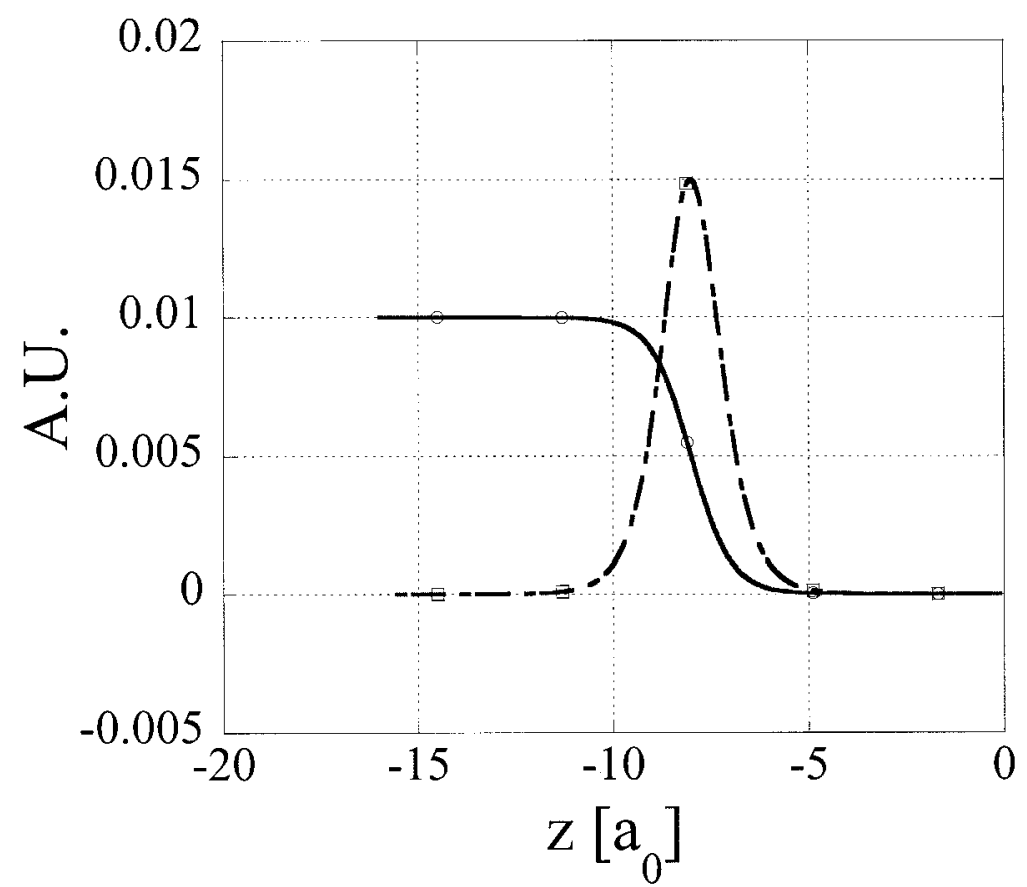

FIGURE 1. The perturbation $v(z)$ (circles) [Eqs. (2) and (12)] and the derived localized electric field $E(z)=e v^{\prime}(z)$ (squares).

$$
\tilde{I}\left(z_{2}, \omega\right)=\int_{-\infty}^{\infty} I\left(z_{2} ; z_{0}, t\right) e^{i \omega t} d t
$$

is the $\mathrm{AC}$ current. A similar equation defines $p(\omega)$. Values of various parameters in these expressions are given in Table I.

By computing the flux $F\left(z_{2}, t\right)=e^{-1} I\left(z_{2}, t\right)$, using TDDFT, the conductance is assessed from the final formula:

$$
\tilde{G}\left(z_{2} ; z_{0}, \omega\right)=g_{0} \frac{\pi \hbar \tilde{F}\left(z_{2}, \omega\right)}{p(\omega) \Delta v}
$$

where $g_{0}=2 e^{2} / h$ is the basic unit of quantum conductance and $\tilde{F}\left(z_{2}, \omega\right)=e^{-1} \tilde{I}\left(z_{2}, \omega\right)$ is the Fourier-transformed flux. In our calculation, we chose the following form for the potential causing the current (see Fig. 1 for its shape):

$$
v(z)=\frac{\Delta v}{1+e^{\alpha z}}
$$

Because our calculation is within the linear response regime, the exact form of the potential does not in principle affect the estimate of the conductance. Yet we consider here an averaged conduc- tance [Eq. (5)] so there is a fairly small dependence of the conductance on this averaging.

\section{Modeling the Metallic Leads}

Any realistic treatment of conductance of a small electronic device must address the specific details of electron distribution and relaxation in the leads $[33,34]$. Following the work of Lang et al. [26-28], we assume the metallic leads can be modeled by jellium plates placed in the proximity of the molecular wire ( $\mathrm{M}$ in Fig. 2).

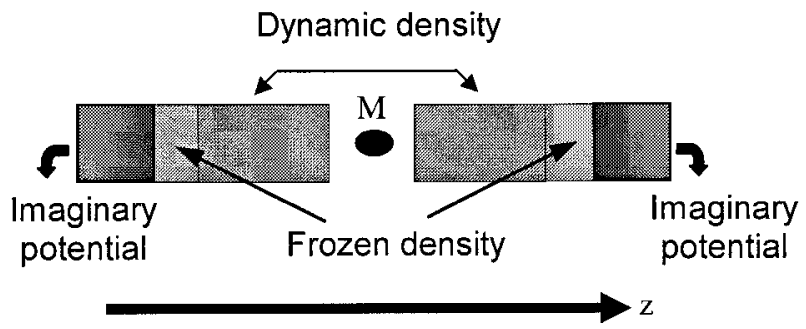

FIGURE 2. The model for molecular wire along the $z$-axis. The molecule $(\mathrm{M})$ is placed between two jellium plates, in which a negative imaginary potential is placed to absorb transported electrons. (See text for further details.) 
TABLE I

Values of various parameters.

\begin{tabular}{|c|c|c|}
\hline Definition & Symbol & Value \\
\hline \multicolumn{3}{|l|}{ Eq. (14) } \\
\hline$a_{0}$ & & $0.0018 E_{h} a_{0}^{-2}$ \\
\hline$z_{L}$ & & $-19 a_{0}$ \\
\hline$z_{R}$ & & $19 a_{0}$ \\
\hline \multicolumn{3}{|l|}{ Eq. (3) } \\
\hline$\sigma$ & & 3 au \\
\hline$T_{o}$ & & 8 au \\
\hline Eq. (4) & $z_{o}$ & $-8 a_{0}$ \\
\hline \multicolumn{3}{|l|}{ Eq. (12) } \\
\hline$\Delta v$ & & $0.01 E_{h}$ \\
\hline$\alpha$ & & $2 a_{0}^{-1}$ \\
\hline Total number of orbitals & $N_{T}$ & 68 \\
\hline $\begin{array}{l}\text { Number of dynamical } \\
\text { orbitals }\end{array}$ & $N_{D}$ & 32 \\
\hline Grid dimensions & $\begin{array}{l}L_{x}=L_{y}, \\
L_{z}\end{array}$ & $10 a_{0}, 65.6 a_{0}$ \\
\hline Grid spacing & $\begin{aligned} \Delta x & =\Delta y \\
& =\Delta z\end{aligned}$ & $0.5 a_{0}$ \\
\hline Jellium density parameter & $r_{s}$ & 3.0 \\
\hline Jellium plates' separation & & $2.2 a_{0}$ \\
\hline C-C distance & & $2.5 a_{0}$ \\
\hline
\end{tabular}

The electron density in the jellium plates is divided into two parts: a frozen density and a dynamical density. The frozen density is located in the part of the plate far from the molecule, whereas the dynamical density includes the molecule. Within the frozen electron density a negative imaginary potential is added to absorb the outgoing electric current, simulating the dissipation mechanism of the macroscopic leads. The reason we introduce a frozen density is because we want to avoid absorbing electron density of the surrounding jellium itself by the imaginary potential: only genuine electric current is to be absorbed. The Hamiltonian used to control the dynamics of the electronic system is:

$$
\hat{H}_{\text {total }}=\hat{H}_{m b}-i \Gamma(\hat{\mathbf{r}})
$$

where $\Gamma(\mathbf{r})$ is a localized potential of the form:

$$
\Gamma(\mathbf{r})=A_{0}\left[\left(z-z_{L}\right)^{2} \theta\left(z_{L}-z\right)+\left(z-z_{R}\right)^{2} \theta\left(z-z_{R}\right)\right]
$$

where $z_{L}$ and $z_{R}$ are deep in the leads. The parameters used in actual calculation are given in Table I.

The imaginary potential is intended to absorb the transmitted electrons. However, as mentioned above, it has an undesired effect: it rapidly absorbs any electronic charge in the jellium leads, even charge not directly involved in the process of transport. To mitigate this artifact, we develop the concept of frozen electron density in the proximity of the absorbing potential. We now discuss how such a frozen density is defined and how the time propagation, within the formalism of TDDFT is performed.

Assume that the molecule-jellium system contains a total of $2 N_{T}$ electrons. The ground-state density, determined by performing a DFT calculation, is partitioned into dynamic and frozen components: $\rho_{T}(\mathbf{r})=\rho_{D}(\mathbf{r})+\rho_{F}(\mathbf{r})$. Both parts are represented as sums of square orthogonal orbitals:

$$
\rho_{D}(\mathbf{r})=\sum_{d=1}^{N_{D}}\left|\varphi_{d}(\mathbf{r})\right|^{2} \quad \rho_{F}(\mathbf{r})=\sum_{l=N_{D}+1}^{N_{T}}\left|\varphi_{l}(\mathbf{r})\right|^{2}
$$

The orbitals $\varphi_{n}(\mathbf{r})$ span the Kohn-Sham occupied space of the total system at its ground state, although they themselves are not Kohn-Sham orbitals. How do we construct this set of orbitals? The key idea is to localize $N_{D}$ states near the molecular wire. This can be done in many ways; we use the following physically motivated method. After obtaining the Kohn-Sham orbitals of the system with all its $2 N_{T}$ electrons, we perform an additional ground-state calculation on a miniaturized system: a system with the same molecule but smaller jellium plates containing only $2 N_{D}$ electrons $\left(N_{D}<\right.$ $\left.N_{T}\right)$. These two calculations produce two sets of Kohn-Sham orbitals: one for the large model and one for the small model of the same system system. The orbitals $\varphi_{n}(\mathbf{r})$ of Eq. (15) are now constructed: they are formed as linear combinations of the Kohn-Sham orbitals of the large model in such a way that the first $N_{D}$ orbitals are as similar as possible to the Kohn-Sham orbitals of the smaller model. Details of the construction are given in the Appendix. As an example, consider the $C_{3}$ molecule between two jellium plates aligned along the $z$-axis (Fig. 2). We adopt the linear configuration of Lang et al. [26], where the C-C distance is $2.5 a_{0}$ and the distance between the positive charged faces of the plates is $2.2 a_{0}$. In both models, large and small, of this system, the jellium plates have the same cross section (area in $x$ and $y$ directions). But the plates in both models differ in their length in the $z$ direction. The large model contains $2 N_{T}=68$ electrons and jellium plates of total positive charge $Q_{J}=56|e|$ that extend from $z=-32$ to $z=32 a_{0}$; the small model contains $2 N_{T}=32$ electrons and its leads extend 


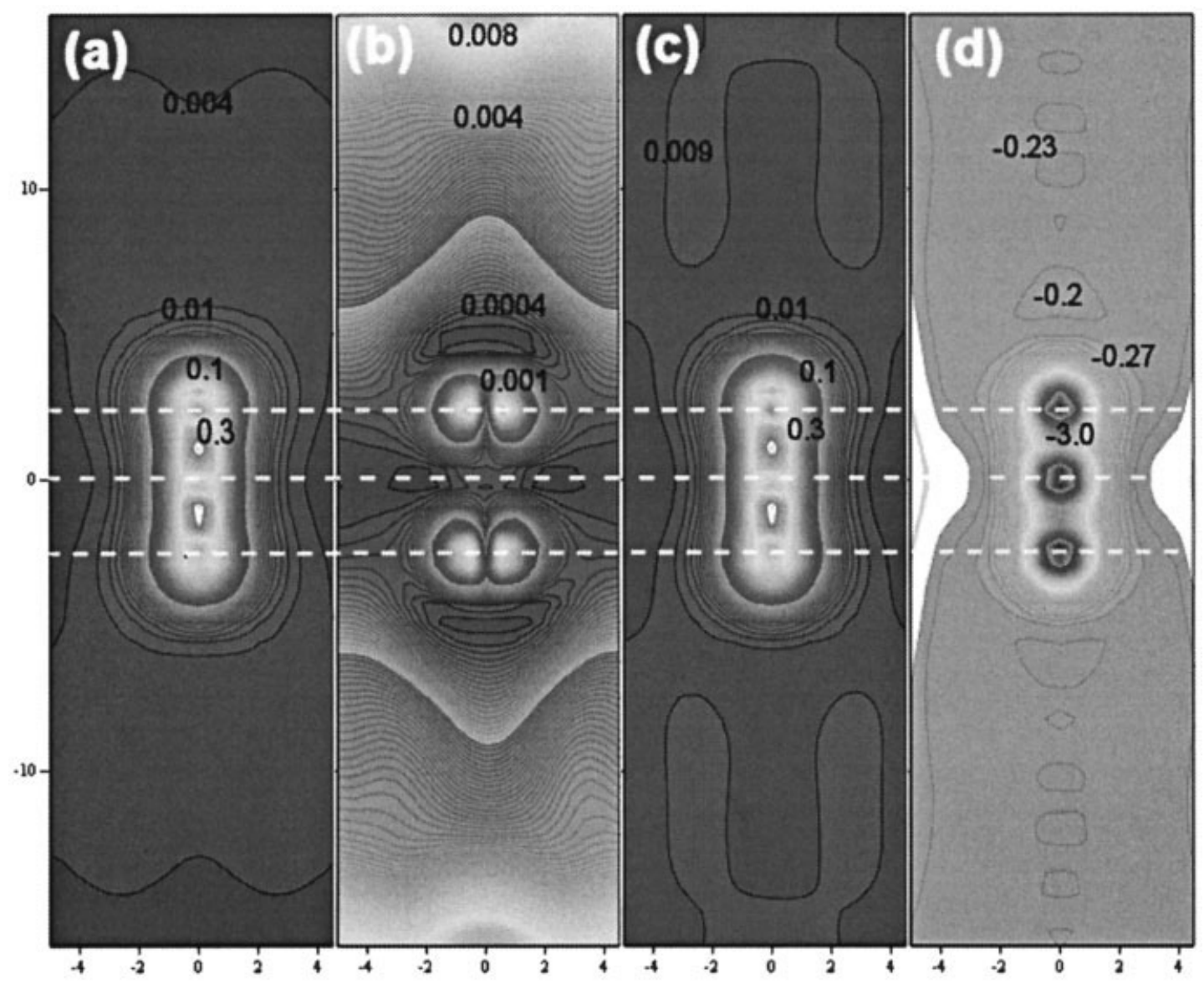

FIGURE 3. Contour plots of the dynamical (a), frozen (b), and total (c) number densities $\left(a_{0}^{-3}\right)$ of the electrons in the $\mathrm{C}_{3}+$ jellium molecular wire. The total number of electrons is 68,32 of which are dynamical and 36 , frozen. Also shown are the contours of the Kohn-Sham effective potential (in $E_{h}$ ) of $C_{3}+$ jellium molecular wire (d). The lengths of the vertical $(z)$ and horizontal axes $(x)$ are indicated in atomic units $a_{0}$. Full length of leads extends from $-32.8 a_{0}$ to $+32.8 a_{0}$.

from $z=-12 z=+12 a_{0}$, involving total positive charge of $Q_{J}=20|e|$. The three types of electron density (dynamic, frozen, and total) of this system are shown in Figure 3. It is seen that the dynamical density, involving $N_{D}=32$ electrons, includes almost all the charge of the $C_{3}$ molecule (12 valence electrons) and the additional charge of the surrounding system (20 electrons from the jellium). Thirty-six electrons are frozen at the far ends of the jellium-comprising the frozen density. Roughly speaking, the dynamic density extends from $z=$ -10 to $z=+10 a_{0}$.

When the system is subject to the time-dependent perturbation $v(z) p(t)$ of Eq. (2), the dynamical density is propagated in time while the frozen density, representing the part of the jellium leads far from the molecule, stays frozen. As the dynamical orbitals are evolved under the Kohn-Sham dynam- ics, they "feel" the frozen density via the Coulomb force and the exchange-correlation potential that this density inspires. But in addition to that, the Pauli principle must be enforced, by demanding the dynamical orbitals be orthogonal to the frozen orbitals. The required TDDFT equations of motion for such a dynamics can be derived from an action principle under the orthogonalization constraint:

$$
i \hbar \dot{\varphi}_{n}(t, \mathbf{r})=\tilde{H} \varphi_{n}(t, \mathbf{r})
$$

where $\tilde{H}\left[\left\{\varphi_{l}\right\}_{l=1}^{N_{T}}\right]$ depends on all the orbitals (dynamical as well as frozen) by:

$$
\tilde{H}\left[\left\{\varphi_{l}\right\}_{l=1}^{N_{T}}\right]=\hat{Q}^{\dagger} H\left[\rho_{T}\right] \hat{Q}
$$

Here $H\left[\rho_{T}\right]$ is the TDDFT effective Hamiltonian, depending on the total density, whereas the projec- 
tion operators $\hat{Q}$ are designed to project out of the frozen space:

$$
\hat{Q}=\hat{1}-\sum_{l=N_{s}+1}^{N_{T}}\left|\psi_{l}\right\rangle\left\langle\psi_{l}\right|
$$

Examining the ground-state Kohn-Sham potential [Fig. 3(d)], a small barrier of about $1 \mathrm{eV}$ is evident as an electron proceeds from the jellium into the molecule. This barrier is low relative to the kinetic energy of highest occupied orbital $(7.1 \mathrm{eV})$. In theories of conduction based on a scattering approach, this is indicative that the molecular wire will exhibit the features of ballistic DC conductance, as indeed reported $[26,27]$. We show that within our calculation the electron-electron interactions within the leads considerably reduce the conductance.

\section{How Is Flux Calculated?}

An important remark concerning the definition of flux $F(z, t)$ used in Eq. (11) is in order. Usually, the flux into a region $\mathfrak{R}$ surrounded by a surface $S(\Re)$ is defined as a surface integral:

$$
F(t)=\iint_{\mathbf{r} \in S(\Re)} \mathbf{J}(\mathbf{r}, t) \cdot d \mathbf{S}
$$

where $\mathbf{J}(\mathbf{r}, t)=(\hbar / \mathrm{m}) \operatorname{Im}\left\{2 \sum_{n=1}^{N_{T}} \psi_{n}^{*}(\mathbf{r}, t) \nabla \psi_{n}(\mathbf{r}, t)\right\}$ is the current density. This definition is not correct for the Hamiltonian of Eq. (17). Instead, the flux into a region $\mathfrak{R}$ should be calculated in a more general way, which we describe now. Assume first a Hamiltonian $\hat{K}(t)=\hat{K}_{r}(T)-i \hat{\Gamma}$ where $\hat{K}_{r}$ is Hermitian but otherwise quite general. Next, define an operator $\Re$ $=\mathfrak{R}(\hat{\mathbf{r}})$ defining the region $\Re$ into which the flux is to be computed, where:

$$
\Re(\mathbf{r})= \begin{cases}1 & \mathbf{r} \in \Re \\ 0 & \text { otherwise }\end{cases}
$$

The flux into $\Re$ is then defined by:

$$
\begin{aligned}
F_{\text {in }}(t) & =\left.\frac{d}{d t} \int\langle\hat{\mathfrak{R}}(\mathbf{r}, t)\rangle d^{3} r\right|_{\text {due to flux }} \\
& =2 i \sum_{n=1}^{N_{e} / 2} \int\left\langle\psi_{n}(\mathbf{r}, t)\left|\left[\hat{K}_{r}(t), \hat{\mathfrak{R}}(\mathbf{r}, t)\right]\right| \psi_{n}(\mathbf{r}, t)\right\rangle d^{3} r
\end{aligned}
$$

This definition leads to Eq. (19) when the Hamiltonian is of the usual Kohn-Sham type: $\hat{K}_{r}(t)=-\left(\hbar^{2} /\right.$ $\left.2 \mathrm{~m}_{e}\right) \nabla^{2}+\mathrm{v}_{s}(\mathbf{r}, t)$. However, Eq. (21) is more general, because it applies to any type of Hamiltonian $\hat{K}_{r}(t)$. In our case, we used Eq. (21) within the Kohn-Sham propagator, taking: $\hat{K}_{r}(t)=Q^{\dagger}\left\{H\left[\rho_{T}\right]+v(z) p(t)\right\} Q$.

\section{TDDFT Evolution}

The equations of motion to be solved are now:

$$
i \hbar \dot{\varphi}_{n}(t)=\hat{K}(t) \varphi_{n}(t) n=1 \ldots N_{D}
$$

with:

$$
\hat{K}=Q^{\dagger}\left(\hat{H}\left[\rho_{T}\right]+v(\hat{z}) p(t)-i \Gamma(\hat{\mathbf{r}})\right) Q
$$

These are nonlinear equations of motion that must be solved to high precision. The method used for their solution has been described elsewhere [32, 35] and we only mention the principle involved. The time axis is partitioned into intervals of $T=0.1$ a.u. Each interval is then sampled by $M$ sampling points $t_{m}(m=1 \ldots M)$. The size of $M$ is determined by the accuracy required. In our calculations we took $M=$ 6. Assuming the knowledge of the states $\varphi_{n}$ at time $m T$, with $m$ an integer, the state of the system at any time within the interval $t \in[m T,(m+1) T]$ is written as:

$$
\varphi_{n}(t)=\sum_{m=1}^{M} \varphi_{n}\left(t_{m}\right) Q_{m}(t)
$$

where $Q_{k}(t)$ are polynomials in time with the collocation property within the interval:

$$
Q_{m}\left(t_{n}\right)=\delta_{n m}
$$

The Kohn-Sham equations, in integral form, are thus transformed to the following discretized equations, which can be solved by iteration:

$$
\psi_{n}\left(t_{m}\right)=\phi-i \frac{T}{\hbar} \sum_{m^{\prime}=0}^{M} \hat{K}\left(\rho_{T}\left(t_{m^{\prime}}\right), t_{m^{\prime}}\right) \psi_{n}\left(t_{m^{\prime}}\right) I_{m^{\prime} m}
$$

with: 


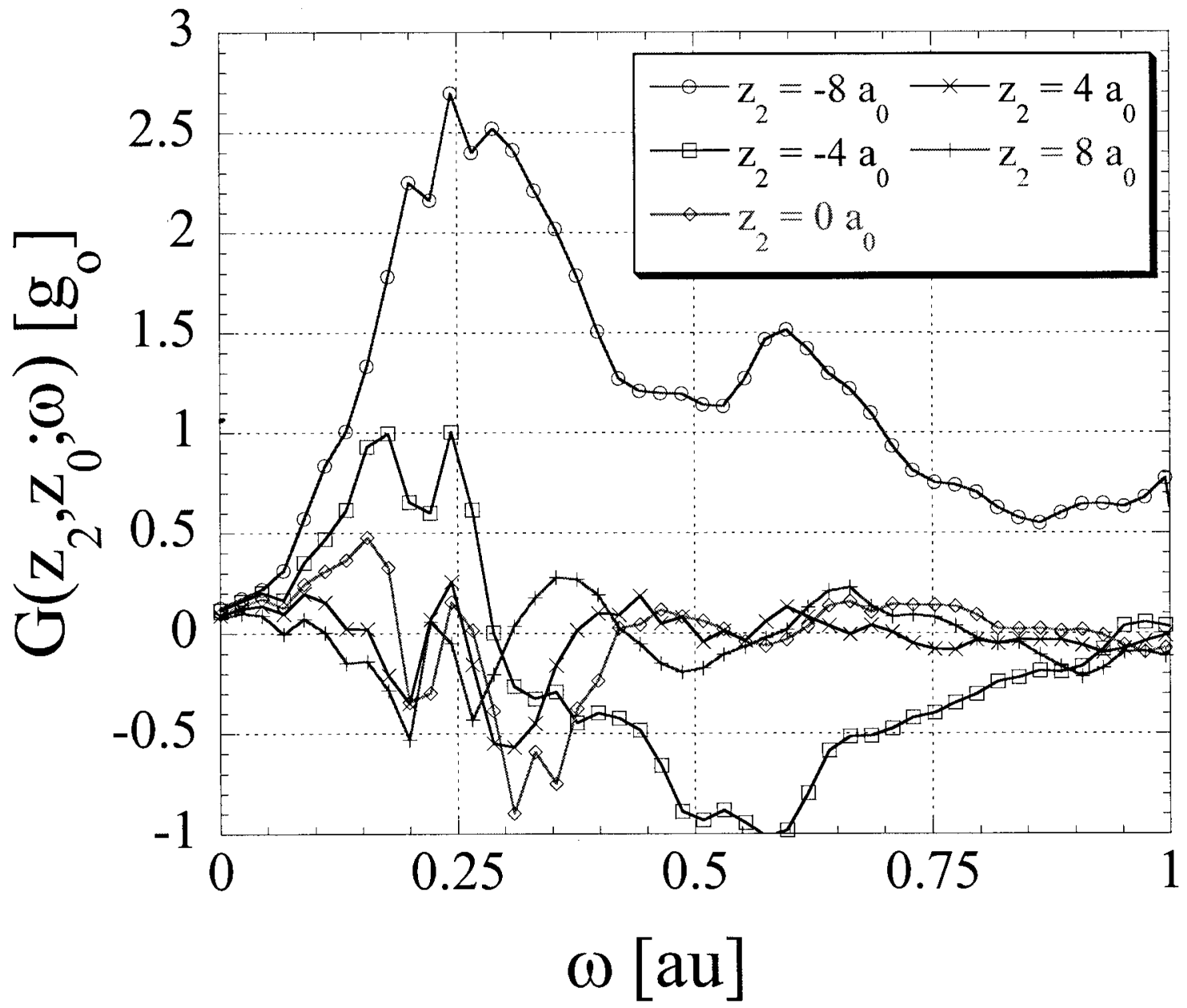

FIGURE 4. The real part of the conductance of $C_{3}+$ jellium. $G\left(z_{2}, z_{0} ; \omega\right)\left(z_{0}=-8 a_{0}\right)$ as calculated at several locations $z_{2}$ along the molecular wire, where the zero of the $z$ axis is the central carbon atom.

$$
I_{l m}=\frac{1}{T} \int_{0}^{t_{l}} Q_{m}(\tau) d \tau
$$

\section{Conductance of $\mathbf{C}_{3}$}

We now apply this method to compute the conductance of the $\mathrm{C}_{3}+$ jellium system. The specific parameters we took are with all summarized in Table I. The current $I(z, \omega)$ at different positions $z$ along the wire is then computed via a TDDFT calculation, using a recently developed numerical propagator [32, 35].

The results of the calculation are shown in Figure 4 , displaying the real part of the conductance. The conductance is a complex number, but only the real (or imaginary) part contains independent data. The
AC results peak at $z$-dependent locations with common peaks at around, $\omega=0.1,0.16,0.26$ a.u. The DC conductance of $\sim 0.15 g_{0}$ is almost $z$-independent.

The DC conductance is smaller by a factor of about 8 than that calculated by Lang et al. $[26,27]$ for the same system. The reason for this discrepancy is probably connected to the different properties assigned to the metallic leads. In the scattering formalism, the leads form a source of energetic electrons, whereas in the current model the coherent dynamics within the leads are explicitly taken into account.

\section{Discussion}

We have presented a new method for computing the conductance of a molecule. The metallic leads are modeled by jellium, whereas the wire is treated 
using pseudopotentials. The far part of the jellium electron density is frozen using the algorithm we outlined. The resulting method allows computation of conductance incorporating electron-electron interaction. Correlation is treated on the TDDFT/ ALDA (adiabatic local density approximation) level and the method includes implicitly the realistic charge distributions. The Kohn-Sham effective potential (Fig. 3) exhibits only a small barrier to conductance, of height $1 \mathrm{eV}$, much less than the kinetic energy of electrons in the highest occupied (Fermi) orbital. Theories based on a scattering approach therefore report a high value of conductance of this system [27] (close to one unit of quantum conductance $\left.g_{0}\right)$. However, the DC conductance we calculated is significantly smaller. We believe the discrepancy stems from the different ways the two models incorporate dissipation into the problem. The method outlined in this article introduces dissipation to absorb outgoing flux. In the scattering approach, both the incoming and the outgoing flux is assigned to dissipative leads, viewed as electron reservoirs [13]. Future investigations will be directed to elucidate the differences between these two mechanisms of conduction.

\section{ACKNOWLEDGMENTS}

This work was supported by the USA Israel Binational Science Foundation. The authors express thanks to M. Ratner, S. Shaik, A. Ben Shaul, and R. Kosloff for their encouragement and assistance. Finally, the Fritz Haber center in the Hebrew University is acknowledged for use of its computational facilities.

\section{Appendix: Dynamic and Frozen Density}

In this appendix we describe the construction of the frozen and dynamic orbitals. This is done by constructing two models of the same molecular wire; one is embedded in the other. The first model contains $N_{D}$ electrons, forming the so-called "dynamical density," whereas the second model has $N_{T}=N_{D}+N_{F}$ electrons, where $N_{F}$ is the number of electrons forming the "frozen density."

As mentioned in the text, the two models are identical except for the extent of the jellium plates. Thus, the difference in the number of electrons is a result of the size of the jellium plates. Now, one performs a ground-state DFT calculation for each of the two models, yielding $N_{D}$ occupied Kohn-Sham orbitals $\psi_{d}(\mathbf{r})$ of the small system $\left(d=1 \ldots N_{D}\right)$ and $N_{T}$ occupied orbitals $\Psi_{t}(\mathbf{r})$ of the larger system $t=$ $1 \ldots N_{T}$.

The charge distribution of the larger system is now partitioned into a dynamical and a frozen part, $\rho_{T}(\mathbf{r})=\rho_{D}(\mathbf{r})+\rho_{F}(\mathbf{r})$ in the following way. First, we define a set of $N_{D}$ orthogonal orbitals $\varphi_{d}(\mathbf{r})(d=$ $1 \ldots N_{D}$ ) that reside completely in the occupied space of the larger system but resemble as closely as possible the occupied orbitals of the small system. Thus, writing:

$$
\varphi_{d}(\mathbf{r})=\sum_{l=1}^{N_{T}} \Psi_{l}(\mathbf{r}) A_{l d}
$$

A rectangular matrix of coefficients $\mathbf{A}=\left(A_{d d^{\prime}}\right)$ is now located, such that the following quantity is minimized:

$$
\sum_{d=1}^{N_{d}}\left\langle\psi_{d}-\varphi_{d} \mid \psi_{d}-\varphi_{d}\right\rangle
$$

under the orthogonality constraint:

$$
\delta_{m m^{\prime}}=\left\langle\varphi_{m} \mid \varphi_{m^{\prime}}\right\rangle \Rightarrow A^{\dagger} A=I_{N_{D} \times N_{D}}
$$

Introducing the $N_{T} \times N_{D}$ overlap matrix $O_{l d}=$ $\left\langle\Psi_{l} \mid \psi_{d}\right\rangle$ and the Lagrange coefficient matrix, $\Lambda_{d d^{\prime}}$, the Lagrangian to be minimized assumes the following form:

$$
L\{A\}=\operatorname{tr}\left[-\left(O^{\dagger} A+A^{\dagger} O\right)+\Lambda A^{\dagger} A\right]
$$

Leading to the conditions:

$$
O=A \Lambda \quad \Lambda=\Lambda^{+}
$$

We invoke the singular-value decomposition theorem [36], according to which, $O=U w V^{+}$where $U$ is a $N_{T} \times N_{D}$ matrix with orthonormal columns $U^{+} U=$ $I_{N_{D} \times N_{D^{\prime}}} V$ is a $N_{D} \times N_{D}$ unitary matrix, $V^{\dagger} V=V V^{\dagger}=$ $I_{N_{D} \times N_{D^{\prime}}}$ and $w$ is a diagonal matrix with real and nonnegative diagonal elements. The decomposition is unique up to row/column permutations of the matrices. The matrix $A$, which minimizes the Lagrangian of Eq. (31), can be written as:

$$
A=U V^{\dagger} \quad \Lambda=V w V^{\dagger}
$$


Thus, the matrix $A$ is immediately given in terms of the overlap matrix $O$. The complement of $\left\{\varphi_{d}\right\}_{d=1}^{N_{D}}$ in the occupied space $\left\{\Psi_{l}\right\}_{l=1}^{N_{T}}$ is described by the orbitals:

$$
\varphi_{f}(\mathbf{r})=\sum_{l=1}^{N_{T}} \Psi_{l}(\mathbf{r}) B_{l f} \quad f=N_{D}+1 \ldots N_{T}
$$

The $N_{T} \times N_{F}$ matrix $B$ of coefficients can be obtained by diagonalizing the Hermitian $N_{T} \times N_{T}$ matrix $U U^{+}$. This matrix must have exactly $N_{F}$ eigenvectors with zero eigenvalues. These eigenvectors can be taken as the columns of $B$.

\section{References}

1. Jortner, J.; Ratner, M. Molecular Electronics; Blackwell Science Inc., Oxford, UK, 1997.

2. Aviram, A.; Ratner, M. A. Chem Phys Lett 1974, 29, 277.

3. Reed, M. A.; Zhou, C.; Muller, C. J.; Burgin, T. P.; Tour, J. M. Science 1997, 278, 252.

4. Collier, C. P.; Wong, E. W.; Belohradsky, M.; Raymo, F. M.; Stoddart, J. F.; Kuekes, P. J.; Williams, R. S.; Heath, J. R. Science 1999, 285, 391.

5. Chen, J.; Reed, M. A.; Rawlett, A. M.; Tour, J. M. Science 1999, 286, 1550.

6. Metzger, R. M. J Mater Chem 2000, 10, 55.

7. Collier, C. P.; Mattersteig, G.; Wong, E. W.; Luo, Y.; Beverly, K.; Sampaio, J.; Raymo, F. M.; Stoddart, J. F.; Heath, J. R. Science 2000, 289, 1172.

8. Rueckes, T.; Kim, K.; Joselevich, E.; Tseng, G. Y.; Cheung, C. L.; Lieber, C. M. Science 2000, 289, 94.

9. Joachim, C.; Gimzewski, J. K.; Aviram, A. Nature 2000, 408, 541.

10. Tour, J. M.; Kozaki, M.; Seminario, J. M. J Am Chem Soc 1998, 120, 8486.

11. Landauer, R. Philos Mag 1970, 21, 863.
12. Nitzan, A. Ann Rev Phys Chem 2001, 52, 681.

13. Datta, S. Electronic Transport in Mesoscopic Systems; Cambridge University Press: Cambridge, 1995.

14. Imry, Y.; Landauer, R. Reviews of Mod Phys 1999, 71, S306.

15. Datta, S.; Tian, W. D.; Hong, S. H.; Reifenberger, R.; Henderson, J. I.; Kubiak, C. P. Phys Rev Lett 1997, 79, 2530.

16. Magoga, M.; Joachim, C. Phys Rev B 1997, 56, 4722.

17. Magoga, M.; Joachim, C. Phys Rev B 1999, 59, 16011.

18. Yaliraki, S.; Kemp, M.; Ratner, M. A. J Am Chem Soc 1999, 121, 3428.

19. Biscarini, F.; Bustamante, C.; Kenkre, V. M. Phys Rev B 1995, 51, 11089.

20. Emberly, E. G.; Kirczenow, G. Phys Rev B 1999, 60, 6028.

21. Hall, L. E.; Reimers, J. R.; Hush, N. S.; Silverbrook, K. J Chem Phys 2000, 112.

22. Mujica, V.; Roitberg, A. E.; Ratner, M. J Chem Phys 2000, 112, 6834.

23. Faglioni, F.; Claypool, C. L.; Lewis, N. S.; Goddard, W. A. III. J Chem Phys 1997, 101, 5996.

24. Larsson, S.; Klimkans, A. J Molec Struct Theochem 1999, 464, 59.

25. Yaliraki, S. N.; Roitberg, A. E.; Gonzalez, C.; Mujica, V.; Ratner, M. A. J Chem Phys 1999, 111, 6997.

26. Lang, N. D.; Avouris, P. Phys Rev Lett 1998, 81, 3515.

27. Lang, N. D.; Avouris, P. Phys Rev Lett 2000, 84, 358.

28. Di Ventra, M.; Pantelides, S. T.; Lang, N. D. Phys Rev Lett 2000, 84, 979.

29. Pretre, A.; Thomas, H.; Buttiker, M. Phys Rev B 1996, 54, 8130.

30. Runge, E.; Gross, E. K. U. Phys Rev Lett 1984, 52, 997.

31. Nakano, A.; Vashishta, P.; Kalia, R. Phys Rev B 1991, 43, 9066.

32. Baer, R.; Gould, R. J Chem Phys 2001, 114, 3385.

33. Buttiker, M.; Imry, Y.; Landauer, R.; Pinhas, S. Phys Rev B 1985, 31, 6207.

34. Kamenev, A.; Kohn, W. Phys Rev B 2001, 6315, 5304.

35. Baer, R. Phys Rev A 2000, 62, 3810.

36. Golub, G. H.; van Loan, C. F. Matrix Computations, 3rd edition; The John Hopkins University Press: Baltimore, 1996. 